\title{
Some new bounds for Simpson's rule involving special functions via harmonic h-convexity
}

Muhammad Uzair Awan ${ }^{\mathrm{a}, *}$, Muhammad Aslam Noor ${ }^{\mathrm{b}, \mathrm{c}}$, Marcela V. Mihaid ${ }^{\mathrm{d}}$, Khalida Inayat Noor ${ }^{\mathrm{e}}$, Awais Gul Khan $^{\mathrm{a}}$

${ }^{a}$ Government College University, Faisalabad, Pakistan.

${ }^{b}$ Department of Mathematics, King Saud University, Riyadh, Saudi Arabia.

${ }^{c}$ Mathematics Department, COMSATS Institute of Information Technology, Islamabad, Pakistan.

${ }^{d}$ Department scientific-methodical sessions, Romanian Mathematical Society-branch Bucharest, Academy Street no. 14, RO-010014, Bucharest, Romania.

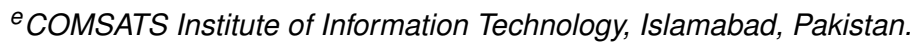

Communicated by J. Pečarić

\begin{abstract}
In this article, we obtain some new bounds for Simpson's rule via harmonic h-convex functions. We also point out some new and known special cases which can be deduced from main results of the article. Some applications to special means are also discussed. (c)2017 All rights reserved.
\end{abstract}

Keywords: Convex functions, bounds, harmonic, differentiable, Simpson inequality. 2010 MSC: 26D15, 26A51.

\section{Introduction and preliminaries}

Theory of convexity plays a pivotal role in different fields of modern mathematics. Consequently in recent decades considerable attention has been given to theory of convexity. As a result the classical concepts of convex sets and convex functions have been extended and generalized in different direction, see [4]. This paper deals with harmonic convexity, so, we define the class of harmonic convex sets.

Definition 1.1 ([20]). A set $K_{h} \subset \mathbb{R} \backslash\{0\}$ is said to be harmonic convex, if

$$
\frac{x y}{\mathrm{t} x+(1-\mathrm{t}) \mathrm{y}} \in \mathrm{K}, \quad \forall x, y \in K, t \in[0,1] \text {. }
$$

Iscan [11] defined the notion of harmonic convex functions as follows.

\footnotetext{
*Corresponding author

Email addresses: awan.uzair@gmail .com (Muhammad Uzair Awan), noormaslam@gmail.com (Muhammad Aslam Noor), marcelamihai58@yahoo.com (Marcela V. Mihai), khalidanoor@hotmail.com (Khalida Inayat Noor), awaisgulkhan@gmail.com (Awais Gul Khan)

doi:10.22436/jnsa.010.04.37
}

Received 2017-01-24 
Definition 1.2 ([11]). Let $K_{h}$ be a harmonic convex set. A function $f: K_{h} \rightarrow \mathbb{R}$ is said to be harmonic convex function, if

$$
f\left(\frac{x y}{t x+(1-t) y}\right) \leqslant(1-t) f(x)+t f(y), \quad \forall x, y \in K_{h}, t \in[0,1] .
$$

Varošanec in [21] introduced an important class of convex functions, which is called as h-convex functions.

Definition 1.3 ([21]). Let $h: J \subseteq \mathbb{R} \rightarrow \mathbb{R}$ be a nonnegative function. We say that $f: I \subseteq \mathbb{R} \rightarrow \mathbb{R}$ is $h$-convex function $(f \in S X(h, I))$, if $f$ is nonnegative and

$$
f(t x+(1-t) y) \leqslant h(t) f(x)+h(1-t) f(y), \quad \forall x, y \in I \text { and } t \in[0,1] .
$$

For $h(t)=t, h(t)=t^{s}, h(t)=\frac{1}{t}, h(t)=1$, and $h(t)=\frac{1}{t^{s}}$, the class of $h$-convex functions reduces to the class of convex functions, s-Breckner convex functions [3], Godunova-Levin functions [9], P-functions [8], and s-Godunova-Levin convex functions [6], respectively. This shows that the class of h-convex functions are quite general and unifying one.

Motivated and inspired by these new classes of convex functions and by the definition of harmonic convex functions, Noor et al. [17] introduced and considered a new class of harmonic convex functions, which is called the harmonic h-convex function.

Definition 1.4 ([17]). A function $f: K_{h} \rightarrow \mathbb{R}$ is said to be harmonic $h$-convex if

$$
f\left(\frac{x y}{t x+(1-t) y}\right) \leqslant h(t) f(y)+h(1-t) f(x), \forall x, y \in I \text { and } t \in[0,1] .
$$

Noor et al. [17] have shown that by taking suitable choice of function $h($.$) one can have some other$ new and known classes of harmonic convex functions. For some recent studies on harmonic $h$-convex functions, see $[13,17]$ and the references therein.

The strong relationship between theory of convexity and theory of inequalities has attracted many researchers, as a result number classical inequalities which were obtained for convex functions have also been extended for other generalizations of convex functions, see [1, 2, 5, 7, 10, 11, 13-19, 22]. Inspired by the ongoing research, in this paper we consider the class of harmonic $h$-convex functions and obtain some new Simpson type inequalities. We discuss some special cases which can be derived from our main results.

From now onward, we take the notation $\mathcal{J}_{h} \subset \mathbb{R} \backslash\{0\}$ be the interval and $\mathcal{J}_{h}^{0}$ be the interior of $\mathcal{J}_{h}$ unless otherwise specified.

\section{Results and discussions}

In this section, we obtain some new bounds for Simpson's rule via harmonic h-convex functions. To derive the main results of this section, we need the following auxiliary result. For the sake of completeness and to convey the main idea we include the proof of this result.

Lemma 2.1 ([18]). Let $f: \mathcal{J}_{h} \rightarrow \mathbb{R}$ be a differentiable function on $\mathcal{J}_{h}^{0}$ and $f \in L_{1}[a, b]$, where $a, b \in \mathcal{J}_{h}$, then

$$
\frac{a b}{b-a} \int_{a}^{b} \frac{f(x)}{x^{2}} d x-\frac{1}{6}\left[f(a)+4 f\left(\frac{2 a b}{a+b}\right)+f(b)\right]=a b(b-a) \int_{0}^{1} \frac{\vartheta(t)}{A_{t}^{2}} f^{\prime}\left(\frac{a b}{A_{t}}\right) d t,
$$

where

$$
\vartheta(t)= \begin{cases}t-\frac{1}{6}, & \text { if } t \in\left[0, \frac{1}{2}\right) \\ t-\frac{5}{6}, & \text { if } t \in\left[\frac{1}{2}, 1\right]\end{cases}
$$

and $\mathrm{A}_{\mathrm{t}}=(1-\mathrm{t}) \mathrm{a}+\mathrm{tb}$. 
Proof. Let

$$
\int_{0}^{1} \frac{\vartheta(t)}{A_{t}^{2}} f^{\prime}\left(\frac{a b}{A_{t}}\right) d t=\int_{0}^{1 / 2} \frac{t-\frac{1}{6}}{A_{t}^{2}} f^{\prime}\left(\frac{a b}{A_{t}}\right) d t+\int_{1 / 2}^{1} \frac{t-\frac{5}{6}}{A_{t}^{2}} f^{\prime}\left(\frac{a b}{A_{t}}\right) d t=G_{1}+G_{2}
$$

Integrating by parts yields

$$
G_{1}=\int_{0}^{1 / 2} \frac{t-\frac{1}{6}}{A_{t}^{2}} f^{\prime}\left(\frac{a b}{A_{t}}\right) d t=\frac{1}{a b(b-a)}\left[-\frac{1}{3} f\left(\frac{2 a b}{a+b}\right)-\frac{1}{6} f(b)+\frac{a b}{b-a} \int_{\frac{2 a b}{a+b}}^{b} \frac{f(x)}{x^{2}} d x\right] .
$$

Similarly

$$
G_{2}=\int_{1 / 2}^{1} \frac{t-\frac{5}{6}}{A_{t}^{2}} f^{\prime}\left(\frac{a b}{A_{t}}\right) d t=\frac{1}{a b(b-a)}\left[-\frac{1}{3} f\left(\frac{2 a b}{a+b}\right)-\frac{1}{6} f(a)+\frac{a b}{b-a} \int_{a}^{\frac{2 a b}{a+b}} \frac{f(x)}{x^{2}} d x\right]
$$

Summation of (2.1), (2.2), and (2.3) and multiplying both sides by $a b(b-a)$ completes the proof.

Now, let us recall some special functions. These functions are extensively used in the development of our coming results. The well-known gamma and beta functions are defined as

$$
\Gamma(x)=\int_{0}^{\infty} e^{-x} t^{x-1} d t, \quad B(x, y)=\int_{0}^{1} t^{x-1}(1-t)^{y-1} d t=\frac{\Gamma(x) \Gamma(y)}{\Gamma(x+y)},
$$

respectively. The integral form of the hypergeometric function is

$$
{ }_{2} F_{1}(x, y ; c ; z)=\frac{1}{B(y, c-y)} \int_{0}^{1} t^{y-1}(1-t)^{c-y-1}(1-z t)^{-x} d t
$$

for $|z|<1, c>y>0$.

Now using Lemma 2.1, we derive some Simpson type inequalities for differentiable harmonic $h$-convex functions.

Theorem 2.2. Let $\mathrm{f}: \mathcal{J}_{\mathrm{h}} \rightarrow \mathbb{R}$ be a differentiable function on $\mathcal{J}_{\mathrm{h}}^{0}$ and $\mathrm{f} \in \mathrm{L}_{1}[\mathrm{a}, \mathrm{b}]$, where $\mathrm{a}, \mathrm{b} \in \mathcal{J}_{\mathrm{h}}$. If $\left|\mathrm{f}^{\prime}\right|^{\mathrm{r}}$ is harmonic h-convex function, then, for $r \geqslant 1$, we have

$$
\begin{aligned}
& \left|\frac{a b}{b-a} \int_{a}^{b} \frac{f(x)}{x^{2}} d x-\frac{1}{6}\left[f(a)+4 f\left(\frac{2 a b}{a+b}\right)+f(b)\right]\right| \\
& \leqslant a b(b-a)\left[P_{1}^{1-\frac{1}{r}}(a, b)\left(\left|f^{\prime}(a)\right|^{r} \phi_{1}(a, b ; h)+\left|f^{\prime}(b)\right|^{r} \phi_{2}(a, b ; h)\right)^{\frac{1}{r}}\right. \\
& \left.\quad+P_{2}^{1-\frac{1}{r}}(a, b)\left(\left|f^{\prime}(a)\right|^{r} \phi_{3}(a, b ; h)+\left|f^{\prime}(b)\right|^{r} \phi_{4}(a, b ; h)\right)^{\frac{1}{r}}\right]
\end{aligned}
$$

where

$$
\begin{aligned}
& P_{1}(a, b)=\frac{(b-3 a)}{6 a\left(b^{2}-a^{2}\right)}+\frac{1}{(b-a)^{2}} \ln \left(\frac{18 a(a+b)}{(5 a+b)^{2}}\right), \\
& P_{2}(a, b)=\frac{(b-3 a)}{6 b\left(b^{2}-a^{2}\right)}+\frac{1}{(b-a)^{2}} \ln \left(\frac{18 b(a+b)}{(a+5 b)^{2}}\right),
\end{aligned}
$$




$$
\begin{aligned}
& \phi_{1}(a, b ; h)=\int_{0}^{1 / 2} \frac{\left|t-\frac{1}{6}\right|}{A_{t}^{2}} h(t) d t, \\
& \phi_{2}(a, b ; h)=\int_{0}^{1 / 2} \frac{\left|t-\frac{1}{6}\right|}{A_{t}^{2}} h(1-t) d t, \\
& \phi_{3}(a, b ; h)=\int_{1 / 2}^{1} \frac{\left|t-\frac{5}{6}\right|}{A_{t}^{2}} h(t) d t,
\end{aligned}
$$

and

$$
\phi_{4}(a, b ; h)=\int_{1 / 2}^{1} \frac{\left|t-\frac{5}{6}\right|}{A_{t}^{2}} h(1-t) d t .
$$

Proof. Using Lemma 2.1, property of modulus, power mean inequality, and the fact that $\left|f^{\prime}\right|^{\mathrm{r}}$ is harmonic h-convex function, we have

$$
\begin{aligned}
& \left|\frac{a b}{b-a} \int_{a}^{b} \frac{f(x)}{x^{2}} d x-\frac{1}{6}\left[f(a)+4 f\left(\frac{2 a b}{a+b}\right)+f(b)\right]\right| \\
& \leqslant a b(b-a)\left[\int_{0}^{1 / 2} \frac{\left|t-\frac{1}{6}\right|}{A_{t}^{2}}\left|f^{\prime}\left(\frac{a b}{A_{t}}\right)\right| d t+\int_{1 / 2}^{1} \frac{\left|t-\frac{5}{6}\right|}{A_{t}^{2}}\left|f^{\prime}\left(\frac{a b}{A_{t}}\right)\right| d t\right] \\
& \leqslant a b(b-a)\left[\left(\int_{0}^{1 / 2} \frac{\left|t-\frac{1}{6}\right|}{A_{t}^{2}} d t\right)^{1-\frac{1}{r}}\left(\int_{0}^{1 / 2} \frac{\left|t-\frac{1}{6}\right|}{A_{t}^{2}}\left|f^{\prime}\left(\frac{a b}{A_{t}}\right)\right|^{r} d t\right)^{\frac{1}{r}}\right. \\
& \left.+\left(\int_{1 / 2}^{1} \frac{\left|t-\frac{5}{6}\right|}{A_{t}^{2}} d t\right)^{1-\frac{1}{r}}\left(\int_{1 / 2}^{1} \frac{\left|t-\frac{5}{6}\right|}{A_{t}^{2}}\left|f^{\prime}\left(\frac{a b}{A_{t}}\right)\right|^{r} d t\right)^{\frac{1}{r}}\right] \\
& \leqslant a b(b-a)\left[\left(\frac{(b-3 a)}{6 a\left(b^{2}-a^{2}\right)}+\frac{1}{(b-a)^{2}} \ln \left(\frac{18 a(a+b)}{(5 a+b)^{2}}\right)\right)^{1-\frac{1}{r}}\right. \\
& \times\left(\int_{0}^{1 / 2} \frac{\left|t-\frac{1}{6}\right|}{A_{t}^{2}}\left\{h(t)\left|f^{\prime}(a)\right|^{r}+h(1-t)\left|f^{\prime}(b)\right|^{r}\right\} d t\right)^{\frac{1}{r}} \\
& +\left(\frac{(b-3 a)}{6 b\left(b^{2}-a^{2}\right)}+\frac{1}{(b-a)^{2}} \ln \left(\frac{18 b(a+b)}{(a+5 b)^{2}}\right)\right)^{1-\frac{1}{r}} \\
& \left.\times\left(\int_{1 / 2}^{1} \frac{\left|t-\frac{5}{6}\right|}{A_{t}^{2}}\left\{h(t)\left|f^{\prime}(a)\right|^{r}+h(1-t)\left|f^{\prime}(b)\right|^{r}\right\} d t\right)^{\frac{1}{r}}\right] \\
& =a b(b-a)\left[\left(\frac{(b-3 a)}{6 a\left(b^{2}-a^{2}\right)}+\frac{1}{(b-a)^{2}} \ln \left(\frac{18 a(a+b)}{(5 a+b)^{2}}\right)\right)^{1-\frac{1}{r}}\right. \\
& \times\left(\left|f^{\prime}(a)\right|^{r} \int_{0}^{1 / 2} \frac{\left|t-\frac{1}{6}\right|}{A_{t}^{2}} h(t) d t+\left|f^{\prime}(b)\right|^{r} \int_{0}^{1 / 2} \frac{\left|t-\frac{1}{6}\right|}{A_{t}^{2}} h(1-t) d t\right)^{\frac{1}{r}}
\end{aligned}
$$




$$
\begin{aligned}
& +\left(\frac{(b-3 a)}{6 b\left(b^{2}-a^{2}\right)}+\frac{1}{(b-a)^{2}} \ln \left(\frac{18 b(a+b)}{(a+5 b)^{2}}\right)\right)^{1-\frac{1}{r}} \\
& \left.\times\left(\left|f^{\prime}(a)\right|^{r} \int_{1 / 2}^{1} \frac{\left|t-\frac{5}{6}\right|}{A_{t}^{2}} h(t) d t+\left|f^{\prime}(b)\right|^{r} \int_{1 / 2}^{1} \frac{\left|t-\frac{5}{6}\right|}{A_{t}^{2}} h(1-t) d t\right)^{\frac{1}{r}}\right] .
\end{aligned}
$$

This completes the proof.

Corollary 2.3. Under the assumptions of Theorem 2.2 if $\mathrm{r}=1$, then, we have

$$
\begin{aligned}
\left|\frac{a b}{b-a} \int_{a}^{b} \frac{f(x)}{x^{2}} d x-\frac{1}{6}\left[f(a)+4 f\left(\frac{2 a b}{a+b}\right)+f(b)\right]\right| \leqslant & a b(b-a)\left[\left|f^{\prime}(a)\right|\left\{\phi_{1}(a, b ; h)+\phi_{3}(a, b ; h)\right\}\right. \\
& \left.+\left|f^{\prime}(b)\right|\left\{\phi_{2}(a, b ; h)+\phi_{4}(a, b ; h)\right\}\right],
\end{aligned}
$$

where $\phi_{1}(a, b ; h), \phi_{2}(a, b ; h), \phi_{3}(a, b ; h)$, and $\phi_{4}(a, b ; h)$ are given by (2.6), (2.7), (2.8), and (2.9) respectively.

We now discuss some special cases of Theorem 2.2.

I. If $h(t)=t$ in Theorem 2.2, then, we have Corollary 10 in [10].

II. If $h(t)=t^{s}$ in Theorem 2.2, then, we have the following.

Corollary 2.4. Let $\mathrm{f}: \mathcal{J}_{\mathrm{h}} \rightarrow \mathbb{R}$ be a differentiable function on $\mathcal{J}_{\mathrm{h}}^{0}$ and $\mathrm{f} \in \mathrm{L}_{1}[\mathrm{a}, \mathrm{b}]$, where $\mathrm{a}, \mathrm{b} \in \mathcal{J}_{\mathrm{h}}$. If $\left|\mathrm{f}^{\prime}\right|^{\mathrm{r}}$ is harmonic s-convex function, then, for $s \in(0,1], r \geqslant 1$, we have

$$
\begin{aligned}
& \left|\frac{a b}{b-a} \int_{a}^{b} \frac{f(x)}{x^{2}} d x-\frac{1}{6}\left[f(a)+4 f\left(\frac{2 a b}{a+b}\right)+f(b)\right]\right| \\
& \leqslant a b(b-a)\left[P_{1}^{1-\frac{1}{r}}(a, b)\left(\left|f^{\prime}(a)\right|^{r} \phi_{1}^{\prime}(a, b ; s)+\left|f^{\prime}(b)\right|^{r} \phi_{2}^{\prime}(a, b ; s)\right)^{\frac{1}{r}}\right. \\
& \left.\quad+P_{2}^{1-\frac{1}{r}}(a, b)\left(\left|f^{\prime}(a)\right|^{r} \phi_{3}^{\prime}(a, b ; s)+\left|f^{\prime}(b)\right|^{r} \phi_{4}^{\prime}(a, b ; s)\right)^{\frac{1}{r}}\right]
\end{aligned}
$$

where $\mathrm{P}_{1}(\mathrm{a}, \mathrm{b}), \mathrm{P}_{2}(\mathrm{a}, \mathrm{b})$ are given by $(2.4),(2.5)$, respectively and

$$
\begin{aligned}
& \mathrm{R}_{1}=\int_{0}^{1 / 2} \frac{\left|\mathrm{t}-\frac{1}{6}\right|}{A_{\mathrm{t}}^{2}} \mathrm{t}^{\mathrm{s}} \mathrm{dt}=\frac{1}{2^{\mathrm{s}+2} \mathrm{a}^{2}}\left[\frac{2}{3^{\mathrm{s}+2}(\mathrm{~s}+1)}{ }_{2} \mathrm{~F}_{1}\left(2, \mathrm{~s}+1 ; \mathrm{s}+2 ; \frac{1}{6}\left(1-\frac{\mathrm{b}}{\mathrm{a}}\right)\right)\right. \\
& -\frac{1}{3^{s+2}(s+2)}{ }_{2} F_{1}\left(2, s+2 ; s+3 ; \frac{1}{6}\left(1-\frac{b}{a}\right)\right) \\
& +\frac{1}{s+2}{ }_{2} F_{1}\left(2, s+2 ; s+3 ; \frac{1}{2}\left(1-\frac{b}{a}\right)\right) \\
& \left.-\frac{1}{3(s+1)}{ }_{2} F_{1}\left(2, s+1 ; s+2 ; \frac{1}{2}\left(1-\frac{b}{a}\right)\right)\right]=\phi_{1}^{\prime}(a, b ; s), \\
& R_{2}=\int_{0}^{1 / 2} \frac{\left|t-\frac{1}{6}\right|}{((1-t) a+t b)^{2}}(1-t)^{s} d t \\
& =\frac{1}{4 \mathrm{a}^{2}}\left[\frac{1}{2}{ }_{2} \mathrm{~F}_{1}\left(2,2 ; 3 ; \frac{1}{2}\left(1-\frac{\mathrm{b}}{\mathrm{a}}\right)\right)-\frac{1}{3}{ }_{2} \mathrm{~F}_{1}\left(2,1 ; 2 ; \frac{1}{2}\left(1-\frac{\mathrm{b}}{\mathrm{a}}\right)\right)\right] \\
& +\frac{1}{18 a^{2}}\left[{ }_{2} F_{1}\left(2,1 ; 2 ; \frac{1}{6}\left(1-\frac{b}{a}\right)\right)-\frac{1}{2}{ }_{2} F_{1}\left(2,2 ; 3 ; \frac{1}{6}\left(1-\frac{b}{a}\right)\right)\right]=\phi_{2}^{\prime}(a, b ; s),
\end{aligned}
$$




$$
\begin{aligned}
\mathrm{R}_{3}= & \int_{1 / 2}^{1} \frac{\left|t-\frac{5}{6}\right|}{((1-t) a+t b)^{2}} t^{s} d t \\
\leqslant & \frac{4}{(a+b)^{2}}\left[\frac{2}{9}{ }_{2} F_{1}\left(2,1 ; 2 ; \frac{2(a-b)}{3(a+b)}\right)-\frac{1}{9}{ }_{2} F_{1}\left(2,2 ; 3 ; \frac{2(a-b)}{3(a+b)}\right)\right. \\
& \left.+\frac{1}{8}{ }_{2} F_{1}\left(2,2 ; 3 ; \frac{a-b}{a+b}\right)-\frac{1}{6}{ }_{2} F_{1}\left(2,1 ; 2 ; \frac{a-b}{a+b}\right)\right]=\phi_{3}^{\prime}(a, b ; s),
\end{aligned}
$$

and

$$
\begin{aligned}
\mathrm{R}_{4}= & \int_{1 / 2}^{1} \frac{\left|t-\frac{5}{6}\right|}{((1-t) a+t b)^{2}}(1-t)^{s} d t \\
\leqslant & \frac{4}{(a+b)^{2}}\left[\frac{2}{9}{ }_{2} F_{1}\left(2,1 ; 2 ; \frac{2(a-b)}{3(a+b)}\right)-\frac{1}{9}{ }_{2} F_{1}\left(2,2 ; 3 ; \frac{2(a-b)}{3(a+b)}\right)\right. \\
& \left.+\frac{1}{8}{ }_{2} F_{1}\left(2,2 ; 3 ; \frac{a-b}{a+b}\right)-\frac{1}{6}{ }_{2} F_{1}\left(2,1 ; 2 ; \frac{a-b}{a+b}\right)\right]=\phi_{4}^{\prime}(a, b ; s) .
\end{aligned}
$$

III. If $h(t)=t^{-s}$ in Theorem 2.2, then, we have the following.

Corollary 2.5. Let $\mathrm{f}: \mathcal{J}_{\mathrm{h}} \rightarrow \mathbb{R}$ be a differentiable function on $\mathcal{J}_{\mathrm{h}}^{0}$ and $\mathrm{f} \in \mathrm{L}_{1}[\mathrm{a}, \mathrm{b}]$, where $\mathrm{a}, \mathrm{b} \in \mathcal{J}_{\mathrm{h}}$. If $\left|\mathrm{f}^{\prime}\right|^{\mathrm{r}}$ is harmonic s-Godunova-Levin convex function, then, for $s \in[0,1], r \geqslant 1$, we have

$$
\begin{aligned}
& \left|\frac{a b}{b-a} \int_{a}^{b} \frac{f(x)}{x^{2}} d x-\frac{1}{6}\left[f(a)+4 f\left(\frac{2 a b}{a+b}\right)+f(b)\right]\right| \\
& \leqslant a b(b-a)\left[P_{1}^{1-\frac{1}{r}}(a, b)\left(\left|f^{\prime}(a)\right|^{r} \phi_{1}^{\prime \prime}(a, b ;-s)+\left|f^{\prime}(b)\right|^{r} \phi_{2}^{\prime \prime}(a, b ;-s)\right)^{\frac{1}{r}}\right. \\
& \left.\quad+P_{2}^{1-\frac{1}{r}}(a, b)\left(\left|f^{\prime}(a)\right|^{r} \phi_{3}^{\prime \prime}(a, b ;-s)+\left|f^{\prime}(b)\right|^{r} \phi_{4}^{\prime \prime}(a, b ;-s)\right)^{\frac{1}{r}}\right],
\end{aligned}
$$

where $P_{1}(a, b), P_{2}(a, b)$ are given by $(2.4),(2.5)$, respectively and

$$
\begin{aligned}
& S_{1}=\int_{0}^{1 / 2} \frac{\left|t-\frac{1}{6}\right|}{A_{t}^{2}} t^{-s} d t \\
& =\frac{1}{2^{2-s} a^{2}}\left[\frac{2}{3^{2-s}(1-s)}{ }_{2} F_{1}\left(2,1-s ; 2-s ; \frac{a-b}{6 a}\right)\right. \\
& -\frac{1}{3^{2-s}(2-s)}{ }_{2} F_{1}\left(2,2-s ; 3-s ; \frac{a-b}{6 a}\right)+\frac{1}{2-s}{ }_{2} F_{1}\left(2,2-s ; 3-s ; \frac{a-b}{2 a}\right) \\
& \left.-\frac{1}{3(1-s)}{ }_{2} F_{1}\left(2,1-s ; 2-s ; \frac{a-b}{2 a}\right)\right]=\phi_{1}^{\prime \prime}(a, b ;-s) \text {, } \\
& S_{2}=\int_{0}^{1 / 2} \frac{\left|t-\frac{1}{6}\right|}{A_{t}^{2}}(1-t)^{-s} d t \\
& \leqslant \frac{2}{4 a^{2}}\left[\frac{1}{2}{ }_{2} F_{1}\left(2,2 ; 3 ; \frac{a-b}{2 a}\right)-\frac{1}{3}{ }_{2} F_{1}\left(2,1 ; 2 ; \frac{a-b}{2 a}\right)\right] \\
& +\frac{2^{1-s}}{36 a^{2}}\left[{ }_{2} F_{1}\left(2,1 ; 2 ; \frac{a-b}{6 a}\right)-\frac{1}{2}{ }_{2} F_{1}\left(2,2 ; 3 ; \frac{a-b}{6 a}\right)\right]=\phi_{2}^{\prime \prime}(a, b ;-s),
\end{aligned}
$$




$$
\begin{aligned}
S_{3}= & \int_{1 / 2}^{1} \frac{\left|t-\frac{5}{6}\right|}{A_{t}^{2}} t^{-s} d t \\
\leqslant & \frac{2^{s+2}}{(a+b)^{2}}\left[\frac{2}{9}{ }_{2} F_{1}\left(2,1 ; 2 ; \frac{2(a-b)}{3(a+b)}\right)-\frac{1}{9}{ }_{2} F_{1}\left(2,2 ; 3 ; \frac{2(a-b)}{3(a+b)}\right)\right. \\
& \left.+\frac{1}{8}{ }_{2} F_{1}\left(2,2 ; 3 ; \frac{a-b}{a+b}\right)-\frac{1}{6}{ }_{2} F_{1}\left(2,1 ; 2 ; \frac{a-b}{a+b}\right)\right]=\phi_{3}^{\prime \prime}(a, b ;-s),
\end{aligned}
$$

and

$$
\begin{aligned}
S_{4}= & \int_{1 / 2}^{1} \frac{\left|t-\frac{5}{6}\right|}{A_{t}^{2}}(1-t)^{-s} d t \\
\leqslant & \frac{56^{s-1}}{a^{2}}\left[\frac{5}{6}{ }_{2} F_{1}\left(2,1 ; 2 ; \frac{5(a-b)}{6 a}\right)-\frac{1}{4}{ }_{2} F_{1}\left(2,1 ; 2 ; \frac{a-b}{2 a}\right)\right] \\
& -\frac{6^{s}}{a^{2}}\left[\frac{25}{72}{ }_{2} F_{1}\left(2,2 ; 3 ; \frac{5(a-b)}{6 a}\right)-\frac{1}{8}{ }_{2} F_{1}\left(2,2 ; 3 ; \frac{a-b}{2 a}\right)\right] \\
& +\frac{1}{(4-s)(3-s) a^{2}}{ }_{2} F_{1}\left(2,2 ; 3-s ; \frac{a-b}{a}\right)-\frac{5}{6(2-s) a^{2}}{ }_{2} F_{1}\left(2,1 ; 2-s ; \frac{a-b}{a}\right) \\
& -\frac{25}{432 a^{2}}{ }_{2} F_{1}\left(2,2 ; 3 ; \frac{5(a-b)}{6 a}\right)+\frac{25}{36 a^{2}}{ }_{2} F_{1}\left(2,1 ; 2 ; \frac{5(a-b)}{6 a}\right)=\phi_{4}^{\prime \prime}(a, b ;-s) .
\end{aligned}
$$

IV. If $h(t)=1$ in Theorem 2.2, then, we have the following.

Corollary 2.6. Let $\mathrm{f}: \mathcal{J}_{\mathrm{h}} \rightarrow \mathbb{R}$ be a differentiable function on $\mathcal{J}_{\mathrm{h}}^{0}$ and $\mathrm{f} \in \mathrm{L}_{\mathrm{l}}[\mathrm{a}, \mathrm{b}]$, where $\mathrm{a}, \mathrm{b} \in \mathcal{J}_{\mathrm{h}}$. If $\left|\mathrm{f}^{\prime}\right|^{\mathrm{r}}$ is harmonic P-function, then, for $\mathrm{r} \geqslant 1$, we have

$$
\left|\frac{a b}{b-a} \int_{a}^{b} \frac{f(x)}{x^{2}} d x-\frac{1}{6}\left[f(a)+4 f\left(\frac{2 a b}{a+b}\right)+f(b)\right]\right| \leqslant a b(b-a)\left[\left(P_{1}(a, b)+P_{2}(a, b)\right)\left(\left|f^{\prime}(a)\right|^{r}+\left|f^{\prime}(b)\right|^{r}\right)^{\frac{1}{r}}\right],
$$

where $P_{1}(a, b)$ and $P_{2}(a, b)$ are given by (2.4) and (2.5), respectively.

Theorem 2.7. Let $\mathrm{f}: \mathcal{J}_{\mathrm{h}} \rightarrow \mathbb{R}$ be a differentiable function on $\mathcal{J}_{\mathrm{h}}^{0}$ and $\mathrm{f} \in \mathrm{L}_{1}[\mathrm{a}, \mathrm{b}]$, where $\mathrm{a}, \mathrm{b} \in \mathcal{J}_{\mathrm{h}}$. If $\left|\mathrm{f}^{\prime}\right|^{\mathrm{r}}$ is harmonic h-convex function, then, for $\mathrm{r}>1, \frac{1}{\mathrm{p}}+\frac{1}{\mathrm{r}}=1$, we have

$$
\begin{aligned}
\left|\frac{a b}{b-a} \int_{a}^{b} \frac{f(x)}{x^{2}} d x-\frac{1}{6}\left[f(a)+4 f\left(\frac{2 a b}{a+b}\right)+f(b)\right]\right| \leqslant & a b(b-a) P^{1 / p}(a, b ; p) \\
& \times\left[\left(\left|f^{\prime}(a)\right|^{r} \theta_{1}(a, b ; r)+\left|f^{\prime}(b)\right|^{r} \theta_{2}(a, b ; r)\right)^{\frac{1}{r}}\right. \\
& \left.+\left(\left|f^{\prime}(a)\right|^{r} \theta_{3}(a, b ; r)+\left|f^{\prime}(b)\right|^{r} \theta_{4}(a, b ; r)\right)^{\frac{1}{r}}\right],
\end{aligned}
$$

where

$$
\begin{gathered}
\mathrm{P}(\mathrm{a}, \mathrm{b} ; \mathrm{p})=\int_{0}^{1 / 2}\left|\mathrm{t}-\frac{1}{6}\right|^{\mathrm{p}} \mathrm{dt}=\int_{1 / 2}^{1}\left|\mathrm{t}-\frac{5}{6}\right|^{\mathrm{p}} \mathrm{dt}=\frac{1}{3^{p+1}(\mathrm{p}+1)}\left(\frac{1}{2^{\mathrm{p}+1}}+1\right), \\
\theta_{1}(\mathrm{a}, \mathrm{b} ; \mathrm{r})=\int_{0}^{1 / 2} \frac{1}{\mathrm{~A}_{\mathrm{t}}^{2 \mathrm{r}}} \mathrm{h}(\mathrm{t}) \mathrm{dt},
\end{gathered}
$$




$$
\begin{aligned}
& \theta_{2}(a, b ; r)=\int_{0}^{1 / 2} \frac{1}{A_{t}^{2 r}} h(1-t) d t \\
& \theta_{3}(a, b ; r)=\int_{1 / 2}^{1} \frac{1}{A_{t}^{2 r}} h(t) d t
\end{aligned}
$$

and

$$
\theta_{4}(a, b ; r)=\int_{1 / 2}^{1} \frac{1}{A_{t}^{2 r}} h(1-t) d t
$$

Proof. Using Lemma 2.1, property of modulus, Hölder inequality, and the fact that $\left|f^{\prime}\right|^{r}$ is harmonic $h$ convex function, we have

$$
\begin{aligned}
& \left|\frac{a b}{b-a} \int_{a}^{b} \frac{f(x)}{x^{2}} d x-\frac{1}{6}\left[f(a)+4 f\left(\frac{2 a b}{a+b}\right)+f(b)\right]\right| \\
& \leqslant a b(b-a)\left[\int_{0}^{1 / 2} \frac{\left|t-\frac{1}{6}\right|}{A_{t}^{2}}\left|f^{\prime}\left(\frac{a b}{((1-t) a+t b)}\right)\right| d t+\int_{1 / 2}^{1} \frac{\left|t-\frac{5}{6}\right|}{((1-t) a+t b)^{2}}\left|f^{\prime}\left(\frac{a b}{((1-t) a+t b)}\right)\right| d t\right] \\
& \leqslant a b(b-a) \times\left[\left(\int_{0}^{1 / 2}\left|t-\frac{1}{6}\right| d t\right)^{\frac{1}{p}}\left(\int_{0}^{1 / 2} \frac{1}{A_{t}^{2 r}}\left|f^{\prime}\left(\frac{a b}{A_{t}}\right)\right|^{r} d t\right)^{\frac{1}{r}}\right. \\
& \left.+\left(\int_{1 / 2}^{1}\left|t-\frac{5}{6}\right| d t\right)^{\frac{1}{p}}\left(\int_{1 / 2}^{1} \frac{1}{A_{t}^{2 r}}\left|f^{\prime}\left(\frac{a b}{A_{t}}\right)\right|^{r} d t\right)^{\frac{1}{r}}\right] \\
& \leqslant a b(b-a) P^{\frac{1}{p}}(a, b ; p) \times\left[\left(\int_{0}^{1 / 2} \frac{1}{A_{t}^{2 r}}\left\{h(t)\left|f^{\prime}(a)\right|^{r}+h(1-t)\left|f^{\prime}(b)\right|^{r}\right\} d t\right)^{\frac{1}{r}}\right. \\
& \left.+\left(\int_{1 / 2}^{1} \frac{1}{A_{t}^{2 r}}\left\{h(t)\left|f^{\prime}(a)\right|^{r}+h(1-t)\left|f^{\prime}(b)\right|^{r}\right\} d t\right)^{\frac{1}{r}}\right] .
\end{aligned}
$$

This completes the proof.

We now discuss some special cases of Theorem 2.7.

I. If $h(t)=t$ in Theorem 2.7, then, we have Corollary 18 of [10].

II. If $h(t)=t^{s}$ in Theorem 2.7, then, we have the following result.

Corollary 2.8. Let $\mathrm{f}: \mathcal{J}_{\mathrm{h}} \rightarrow \mathbb{R}$ be a differentiable function on $\mathcal{J}_{\mathrm{h}}^{0}$ and $\mathrm{f} \in \mathrm{L}_{1}[\mathrm{a}, \mathrm{b}]$, where $\mathrm{a}, \mathrm{b} \in \mathcal{J}_{\mathrm{h}}$. If $\left|\mathrm{f}^{\prime}\right|^{\mathrm{r}}$ is harmonic s-convex function, then, for $s \in(0,1], r>1, \frac{1}{p}+\frac{1}{r}=1$, we have

$$
\begin{aligned}
& \left|\frac{a b}{b-a} \int_{a}^{b} \frac{f(x)}{x^{2}} d x-\frac{1}{6}\left[f(a)+4 f\left(\frac{2 a b}{a+b}\right)+f(b)\right]\right| \\
& \leqslant a b(b-a) P^{\frac{1}{p}}(a, b ; p)\left[\left(\left|f^{\prime}(a)\right|^{r} \theta_{1}^{\prime}(a, b ; s)+\left|f^{\prime}(b)\right|^{r} \theta_{2}^{\prime}(a, b ; s)\right)^{\frac{1}{r}}\right. \\
& \left.\quad+\left(\left|f^{\prime}(a)\right|^{r} \theta_{3}^{\prime}(a, b ; s)+\left|f^{\prime}(b)\right|^{r} \theta_{4}^{\prime}(a, b ; s)\right)^{\frac{1}{r}}\right],
\end{aligned}
$$


where $\mathrm{P}(\mathrm{a}, \mathrm{b} ; \mathrm{p})$ is given by $(2.14)$ and

$$
\begin{aligned}
\mathrm{T}_{1}= & \int_{0}^{1 / 2} \frac{1}{A_{\mathrm{t}}^{2 r}} \mathrm{t}^{s} \mathrm{dt}=\frac{1}{2^{s+1} \mathrm{a}^{2 r}(s+1)}{ }_{2} \mathrm{~F}_{1}\left(2 \mathrm{r}, \mathrm{s}+1 ; s+2 ; \frac{\mathrm{a}-\mathrm{b}}{2 \mathrm{a}}\right)=\theta_{1}^{\prime}(\mathrm{a}, \mathrm{b} ; \mathrm{s}), \\
\mathrm{T}_{2}= & \int_{0}^{1 / 2} \frac{1}{A_{\mathrm{t}}^{2 r}}(1-\mathrm{t})^{s} \mathrm{dt} \leqslant \frac{1}{2 \mathrm{a}^{2 r}}{ }_{2} \mathrm{~F}_{1}\left(2 \mathrm{r}, 1 ; 2 ; \frac{\mathrm{a}-\mathrm{b}}{2 \mathrm{a}}\right)=\theta_{2}^{\prime}(\mathrm{a}, \mathrm{b} ; \mathrm{s}), \\
\mathrm{T}_{3}= & \int_{1 / 2}^{1} \frac{1}{A_{\mathrm{t}}^{2 r}} \mathrm{t}^{\mathrm{s}} \mathrm{dt}=\frac{1}{\mathrm{a}^{2 r}(\mathrm{~s}+1)}\left[{ }_{2} \mathrm{~F}_{1}\left(2 \mathrm{r}, \mathrm{s}+1 ; s+2 ; \frac{\mathrm{a}-\mathrm{b}}{\mathrm{a}}\right)\right. \\
& \left.-\frac{1}{2^{s+1}}{ }_{2} \mathrm{~F}_{1}\left(2 \mathrm{r}, \mathrm{s}+1 ; s+2 ; \frac{\mathrm{a}-\mathrm{b}}{2 \mathrm{a}}\right)\right]=\theta_{3}^{\prime}(\mathrm{a}, \mathrm{b} ; \mathrm{s}),
\end{aligned}
$$

and

$$
\mathrm{T}_{4}=\int_{1 / 2}^{1} \frac{1}{A_{\mathrm{t}}^{2 r}}(1-\mathrm{t})^{\mathrm{s}} \mathrm{dt} \leqslant \frac{1}{2 \mathrm{a}^{2 \mathrm{r}}}\left[{ }_{2} \mathrm{~F}_{1}\left(2 \mathrm{r}, 1 ; 2 ; \frac{\mathrm{a}-\mathrm{b}}{\mathrm{a}}\right)-\frac{1}{2}{ }_{2} \mathrm{~F}_{1}\left(2 \mathrm{r}, 1 ; 2 ; \frac{\mathrm{a}-\mathrm{b}}{2 \mathrm{a}}\right)\right]=\theta_{4}^{\prime}(\mathrm{a}, \mathrm{b} ; \mathrm{s}) .
$$

III. If $h(t)=t^{-s}$ in Theorem 2.7, then, we have the following result.

Corollary 2.9. Let $\mathrm{f}: \mathcal{J}_{\mathrm{h}} \rightarrow \mathbb{R}$ be a differentiable function on $\mathcal{J}_{\mathrm{h}}^{0}$ and $\mathrm{f} \in \mathrm{L}_{\mathrm{l}}[\mathrm{a}, \mathrm{b}]$, where $\mathrm{a}, \mathrm{b} \in \mathcal{J}_{\mathrm{h}}$. If $\left|\mathrm{f}^{\prime}\right|^{\mathrm{r}}$ is harmonic s-Godunova-Levin convex function, then, for $s \in[0,1], r>1, \frac{1}{p}+\frac{1}{r}=1$, we have

$$
\begin{aligned}
& \left|\frac{a b}{b-a} \int_{a}^{b} \frac{f(x)}{x^{2}} d x-\frac{1}{6}\left[f(a)+4 f\left(\frac{2 a b}{a+b}\right)+f(b)\right]\right| \\
& \leqslant a b(b-a) P^{\frac{1}{p}}(a, b ; p)\left[\left(\left|f^{\prime}(a)\right|^{r} \theta_{1}^{\prime \prime}(a, b, ; t ;-s)+\left|f^{\prime}(b)\right|^{r} \theta_{2}^{\prime \prime}(a, b, ; t ;-s)\right)^{\frac{1}{r}}\right. \\
& \left.\quad+\left(\left|f^{\prime}(a)\right|^{r} \theta_{3}^{\prime \prime}(a, b, ; t ;-s)+\left|f^{\prime}(b)\right|^{r} \theta_{4}^{\prime \prime}(a, b, ; t ;-s)\right)^{\frac{1}{r}}\right],
\end{aligned}
$$

where $\mathrm{P}(\mathrm{a}, \mathrm{b} ; \mathrm{p})$ is given by $(2.14)$, and

$$
\begin{aligned}
\mathrm{U}_{1}= & \int_{0}^{1 / 2} \frac{1}{A_{\mathrm{t}}^{2 r}} \mathrm{t}^{-s} \mathrm{dt}=\frac{1}{2^{1-s} \mathrm{a}^{2 \mathrm{r}}(1-\mathrm{s})}{ }_{2} \mathrm{~F}_{1}\left(2 \mathrm{r}, 1-\mathrm{s} ; 2-\mathrm{s} ; \frac{\mathrm{a}-\mathrm{b}}{2 \mathrm{a}}\right)=\theta_{1}^{\prime \prime}(\mathrm{a}, \mathrm{b} ;-\mathrm{s}), \\
\mathrm{U}_{2}= & \int_{0}^{1 / 2} \frac{1}{\mathrm{~A}_{\mathrm{t}}^{2 r}}(1-\mathrm{t})^{-s} \mathrm{dt} \leqslant \frac{1}{2 \mathrm{a}^{2 \mathrm{r}}}{ }_{2} \mathrm{~F}_{1}\left(2 \mathrm{r}, 1 ; 2 ; \frac{\mathrm{a}-\mathrm{b}}{2 \mathrm{a}}\right)=\theta_{2}^{\prime \prime}(\mathrm{a}, \mathrm{b} ;-\mathrm{s}), \\
\mathrm{U}_{3}= & \int_{1 / 2}^{1} \frac{1}{\mathrm{~A}_{\mathrm{t}}^{2 \mathrm{r}}} \mathrm{t}^{-s} \mathrm{dt}=\frac{1}{\mathrm{a}^{2 \mathrm{r}}(1-\mathrm{s})}\left[{ }_{2} \mathrm{~F}_{1}\left(2 \mathrm{r}, 1-\mathrm{s} ; 2-\mathrm{s} ; \frac{\mathrm{a}-\mathrm{b}}{\mathrm{a}}\right)\right. \\
& \left.-\frac{1}{2^{1-s}}{ }_{2} \mathrm{~F}_{1}\left(2 \mathrm{r}, 1-\mathrm{s} ; 2-\mathrm{s} ; \frac{\mathrm{a}-\mathrm{b}}{2 \mathrm{a}}\right)\right]=\theta_{3}^{\prime \prime}(\mathrm{a}, \mathrm{b} ;-\mathrm{s}),
\end{aligned}
$$

and

$$
\mathrm{U}_{4}=\int_{1 / 2}^{1} \frac{1}{A_{\mathrm{t}}^{2 r}}(1-\mathrm{t})^{-\mathrm{s}} \mathrm{dt} \leqslant \frac{1}{2 \mathrm{a}^{2 r}}\left[{ }_{2} \mathrm{~F}_{1}\left(2 \mathrm{r}, 1 ; 2 ; \frac{\mathrm{a}-\mathrm{b}}{\mathrm{a}}\right)-\frac{1}{2}{ }_{2} \mathrm{~F}_{1}\left(2 \mathrm{r}, 1 ; 2 ; \frac{\mathrm{a}-\mathrm{b}}{2 \mathrm{a}}\right)\right]=\theta_{4}^{\prime \prime}(\mathrm{a}, \mathrm{b} ;-\mathrm{s}) .
$$


IV. If $h(t)=1$ in Theorem 2.7, then, we have the following corollary.

Corollary 2.10. Let $\mathrm{f}: \mathcal{J}_{\mathrm{h}} \rightarrow \mathbb{R}$ be a differentiable function on $\mathcal{J}_{h}^{0}$ and $\mathrm{f} \in \mathrm{L}_{1}[\mathrm{a}, \mathrm{b}]$, where $\mathrm{a}, \mathrm{b} \in \mathcal{J}_{\mathrm{h}}$. If $\left|\mathrm{f}^{\prime}\right|^{\mathrm{r}}$ is harmonic P-convex function, then, for, $r>1, \frac{1}{p}+\frac{1}{r}=1$, we have

$$
\begin{aligned}
& \left|\frac{a b}{b-a} \int_{a}^{b} \frac{f(x)}{x^{2}} d x-\frac{1}{6}\left[f(a)+4 f\left(\frac{2 a b}{a+b}\right)+f(b)\right]\right| \\
& \leqslant a b(b-a) P^{\frac{1}{p}}(a, b ; p)\left(\theta_{1}^{\frac{1}{r}}(a, b ; r)+\theta_{2}^{\frac{1}{r}}(a, b ; r)\right)\left(\left|f^{\prime}(a)\right|^{r}+\left|f^{\prime}(b)\right|^{r}\right)^{\frac{1}{r}},
\end{aligned}
$$

where $\mathrm{P}(\mathrm{a}, \mathrm{b} ; \mathrm{p})$ is given by $(2.14)$, and

$$
\begin{aligned}
& \theta_{1}(a, b ; r)=\int_{0}^{1 / 2} \frac{1}{A_{t}^{2 r}} d t=\frac{1}{2 a^{2 r}}{ }_{2} F_{1}\left(2 r, 1 ; 2 ; \frac{a-b}{2 a}\right), \\
& \theta_{2}(a, b ; r)=\int_{1 / 2}^{1} \frac{1}{A_{t}^{2 r}} d t=\frac{1}{2 a^{2 r}}\left[2 F_{1}\left(2 r, 1 ; 2 ; \frac{a-b}{a}\right)-2 F_{1}\left(2 r, 1 ; 2 ; \frac{a-b}{2 a}\right)\right] .
\end{aligned}
$$

\section{Applications}

Let us recall means for arbitrary real numbers $\alpha, \beta$ where $\alpha \neq \beta$. These means are frequently used in numerical approximation.

1. Arithmetic mean:

$$
A(\alpha, \beta):=\frac{\alpha+\beta}{2}, \quad \alpha, \beta \in \mathbb{R}^{+}
$$

2. Harmonic mean:

$$
H(\alpha, \beta):=\frac{2 \alpha \beta}{\alpha+\beta}, \quad \alpha, \beta \in \mathbb{R}^{+}
$$

3. Logarithmic mean:

$$
\mathrm{L}(\alpha, \beta):=\frac{\beta-\alpha}{\ln \beta-\ln \alpha}, \quad \alpha, \beta \in \mathbb{R}^{+} .
$$

4. Generalized logarithmic mean:

$$
L_{n}(\alpha, \beta):=\left(\frac{\beta^{n+1}-\alpha^{n+1}}{(n+1)(b-a)}\right)^{\frac{1}{n}}, \quad n \in\{-1,0\}, \alpha, \beta \in \mathbb{R}^{+} .
$$

It is known that $H(\alpha, \beta) \leqslant L(\alpha, \beta) \leqslant A(\alpha, \beta)$ and also it is known ([12]) that a function $f:(0,1] \rightarrow(0,1]$, $f(x)=\chi^{s}$ is harmonically s-convex function.

Proposition 3.1. Let $0<\mathrm{a}<\mathrm{b}$ and $\mathrm{s} \in(0,1)$, then

$$
\begin{aligned}
\left|a b L_{s-1}(a, b)-\frac{1}{3}\left[A^{s}(a, b)+2 H^{s}(a, b)\right]\right| \leqslant & |s| a b(b-a)\left[P_{1}^{1-\frac{1}{r}}(a, b)\left(\left|a^{s-1}\right|^{r} \phi_{1}^{\prime}(a, b ; s)+\left|b^{s-1}\right|^{r} \phi_{2}^{\prime}(a, b ; s)\right)^{\frac{1}{r}}\right. \\
& \left.+P_{2}^{1-\frac{1}{r}}(a, b)\left(\left|a^{s-1}\right|^{r} \phi_{3}^{\prime}(a, b ; s)+\left|b^{s-1}\right|^{r} \phi_{4}^{\prime}(a, b ; s)\right)^{\frac{1}{r}}\right],
\end{aligned}
$$

where $\mathrm{P}_{1}^{1-\frac{1}{\mathrm{r}}}(\mathrm{a}, \mathrm{b}), \mathrm{P}_{2}^{1-\frac{1}{\mathrm{r}}}(\mathrm{a}, \mathrm{b}), \phi_{1}^{\prime}(\mathrm{a}, \mathrm{b} ; \mathrm{s}), \phi_{2}^{\prime}(\mathrm{a}, \mathrm{b} ; \mathrm{s}), \phi_{3}^{\prime}(\mathrm{a}, \mathrm{b} ; \mathrm{s})$, and $\phi_{4}^{\prime}(\mathrm{a}, \mathrm{b} ; \mathrm{s})$ are given by $(2.4),(2.5),(2.10)$, (2.11), (2.12), and (2.13), respectively. 
Proof. The assertion follows directly from Corollary 2.4 applied to the harmonically s-convex function $f:(0,1] \rightarrow(0,1], f(x)=x^{s}$.

Proposition 3.2. Let $0<\mathrm{a}<\mathrm{b}$ and $\mathrm{s} \in(0,1)$, we have

$$
\begin{aligned}
\left|a b L_{s-1}(a, b)-\frac{1}{3}\left[A^{s}(a, b)+2 H^{s}(a, b)\right]\right| \leqslant & |s| a b(b-a) P^{\frac{1}{p}}(a, b ; p) \\
\times & {\left[\left(|a|^{r(s-1)} \theta_{1}^{\prime}(a, b ; s)+|b|^{r(r-1)} \theta_{2}^{\prime}(a, b ; s)\right)^{\frac{1}{r}}\right.} \\
& \left.+\left(|a|^{r(s-1)} \theta_{3}^{\prime}(a, b ; s)+|b|^{r(s-1)} \theta_{4}^{\prime}(a, b ; s)\right)^{\frac{1}{r}}\right],
\end{aligned}
$$

where $P(a, b ; p), \theta_{1}^{\prime}(a, b ; s), \theta_{2}^{\prime}(a, b ; s), \theta_{3}^{\prime}(a, b ; s)$, and $\theta_{4}^{\prime}(a, b ; s)$ are given by (2.14), (2.15), (2.16), (2.17), and (2.18), respectively.

Proof. The assertion follows directly from Corollary 2.8 applied to the harmonically s-convex function $f:(0,1] \rightarrow(0,1], f(x)=x^{s}$.

\section{Conclusion}

In this article, we have obtained several new bounds for Simpson's rule via harmonic $h$-convex functions. We have also discussed numerous special cases which are naturally included in our main results. In the last section, we have given some applications to special means of real numbers. These new results can be used where bounds for natural phenomena described by integrals such as mechanical work are frequently required. These results are also helpful in the field of numerical analysis where error analysis is required. Thus it is expected that the results obtained in this article may stimulate further research in this field.

\section{Acknowledgment}

Authors would like express their gratitude to the editor and referee for their constructive comments and suggestions. Authors are pleased to acknowledge the "support of Distinguished Scientist Fellowship Program (DSFP), King Saud University, Riyadh, Saudi Arabia".

\section{References}

[1] M. Alomari, M. Darus, On some inequalities of Simpson-type via quasi-convex functions and applications, Transylv. J. Math. Mech., 2 (2010), 15-24. 1

[2] M. Alomari, M. Darus, S. S. Dragomir, New inequalities of Simpson's type for s-convex functions with applications, RGMIA Res. Rep. Coll., 12 (2009), 18 pages. 1

[3] W. W. Breckner, Stetigkeitsaussagen für eine Klasse verallgemeinerter konvexer Funktionen in topologischen linearen Räumen, (German) Publ. Inst. Math. (Beograd) (N.S.), 23(37) (1978), 13-20. 1

[4] G. Cristescu, L. Lupşa, Non-connected convexities and applications, Applied Optimization, Kluwer Academic Publishers, Dordrecht, (2002). 1

[5] G. Cristescu, M. A. Noor, M. U. Awan, Bounds of the second degree cumulative frontier gaps of functions with generalized convexity, Carpathian J. Math., 31 (2015), 173-180. 1

[6] S. S. Dragomir, Inequalities of Hermite-Hadamard type for h-convex functions on linear spaces, RGMIA Res. Rep. Coll., Article 72, (2013). f 1

[7] S. S. Dragomir, R. P. Agarwal, P. Cerone, On Simpson's inequality and applications, J. Inequal. Appl., 5 (2000), 533-579. 1

[8] S. S. Dragomir, J. Pečarić, L. E. Persson, Some inequalities of Hadamard type, Soochow J. Math., 21 (1995), 335-341. 1

[9] E. K. Godunova, V. I. Levin, Inequalities for functions of a broad class that contains convex, monotone and some other forms of functions, (Russian) Numerical mathematics and mathematical physics (Russian), Moskov. Gos. Ped. Inst., Moscow, 166 (1985), 138-142. 1 
[10] İ. İşcan, Hermite-Hadamard and Simpson-like type inequalities for differentiable harmonically convex functions, J. Math., 2014 (2014), 10 pages. 1, 2, 2

[11] İ. İşcan, Hermite-Hadamard type inequalities for harmonically convex functions, Hacet. J. Math. Stat., 43 (2014), $935-942$. $1,1.2,1$

[12] İ. İşcan, Ostrowski type inequalities for harmonically s-convex functions, Konuralp J. Math., 3 (2015), 63-74. 3

[13] M. V. Mihai, M. A. Noor, K. I. Noor, M. U. Awan, Some integral inequalities for harmonic h-convex functions involving hypergeometric functions, Appl. Math. Comput., 252 (2015), 257-262. 1

[14] M. A. Noor, G. Cristescu, M. U. Awan, Generalized fractional Hermite-Hadamard inequalities for twice differentiable s-convex functions, Filomat, 29 (2015), 807-815.

[15] M. A. Noor, K. I. Noor, M. U. Awan, Some characterizations of harmonically log-convex functions, Proc. Jangjeon Math. Soc., 17 (2014), 51-61.

[16] M. A. Noor, K. I. Noor, M. U. Awan, Integral inequalities for coordinated harmonically convex functions, Complex Var. Elliptic Equ., 60 (2015), 776-786.

[17] M. A. Noor, K. I. Noor, M. U. Awan, S. Costache, Some integral inequalities for harmonically h-convex functions, Politehn. Univ. Bucharest Sci. Bull. Ser. A Appl. Math. Phys., 77 (2015), 5-16. 1, 1.4

[18] J. Park, Hermite-Hadamard-like and Simpson-like type inequalities for harmonically convex functions, Int. J. Math. Anal., 8 (2014), 1321-1337. 2.1

[19] M. Z. Sarikaya, E. Set, M. E. Ozdemir, On new inequalities of Simpson's type for s-convex functions, Comput. Math. Appl., 60 (2010), 2191-2199. 1

[20] H.-N. Shi, J. Zhang, Some new judgement theorems of Schur geometric and Schur harmonic convexities for a class of symmetric functions, J. Inequal. Appl., 2013 (2013), 9 pages. 1.1

[21] S. Varošanec, On h-convexity, J. Math. Anal. Appl., 326 (2007), 303-311. 1, 1.3

[22] Y. Wang, S.-H. Wang, F. Qi, Simpson type integral inequalities in which the power of the absolute value of the first derivative of the integrand is s-preinvex, Facta Univ. Ser. Math. Inform., 28 (2013), 151-159. 1 